\title{
Desenvolvimento de um veículo espacial didático - Sonda Rover
}

\author{
Maria Julia Fischer \\ Mateus Muller Ribeiro \\ Ana Carolina Luciani Corrêa \\ Edson Luiz Zuchi \\ Daniel Veloso Batschauer \\ gfig.ifsc@gmail.com \\ GFIG - Grupo de Foguetes do IFSC Gaspar
}

\author{
Leonardo Ronald Perin Rauta \\ Maurício Justino \\ leonardo.rauta@ifsc.edu.br \\ mauricio.justino@ifsc.edu.br \\ GFIG - Grupo de Foguetes do IFSC Gaspar \\ Instituto Federal de Santa Catarina - Câmpus Gaspar \\ Gaspar, Santa Catarina
}

\begin{abstract}
RESUMO
Space exploration has been going on for many years and, in recent years, has expanded due to the rare metals and compounds in the terrestrial environment. However, before sending machines, equipment and even human beings to explore the stars of the solar system, it is necessary to carry out an analysis and recognition of the environment of that star. Thus came the probes, remotely controlled equipment to explore stars in the solar system. This work presents the development of a low-cost rover probe to be used in a school environment and to increase students' interest in entering the STEAMs. The rover presented here had a total cost of 4,231.60, has a PVC structure, a camera for WiFi transmission of what you are watching and a remote control to control the rover.
\end{abstract}

\section{KEYWORDS}

Veículo, Sonda, Espacial, Rover, Sistemas embarcados, Exploração, Interplanetária

\section{INTRODUÇÃO}

Nos últimos anos o interesse em explorar outros planetas têm aumentado consideravelmente. Diversas agências espaciais e empresas privadas estão desenvolvendo os mais diversos equipamentos para mapeamento e exploração desse planetas. Estados Unidos, Rússia e China já possuem veículos espaciais na Lua e em Marte [1], além de outros explorando os confins do universo. Outros países, principalmente Israel e Índia, também estão iniciando a exploração espacial por meio do envio de veículos espaciais [2].

Essa corrida espacial teve início ainda na guerra fria, mas tem aumentado nos últimos anos devido à evolução tecnológica e a necessidade das nações afirmarem uma posição de destaque tecnológico mundial, explorando, o que até então era considerado inexplorável, outros planetas e luas.

A exploração planetária geralmente é feita utilizando sondas espaciais, que nada mais são do que naves e veículos não tripuladas. Dentro das sondas, existe uma classificação quanto à missão para que foi designada, destacando-se as sondas de sobrevoo, como o helicóptero que será enviado para Marte [3], tem como objetivo sobrevoar o planeta vermelho e adquirir informações sobre ele; sonda orbitadora, que funciona como um satélite ao redor do astro; sonda lander, como a sonda OSIRIS-REx [4], que pousou no cometa Bennu para aquisição de material; sonda de impacto, projetadas para colidir com o astro e analisar esse impacto; e as sondas rovers, que pousam e se locomovem na superfície do astro [5]. Esse trabalho tem foco em sonda rover, um veículo capaz de andar em diferentes terrenos e superfícies.

Essas sondas rover possuem funções e objetivos a serem cumpridos durante sua missão espacial. Geralmente essas missões consistem em explorar o relevo, clima, solo, entre outras.

Porém, um veículo que possa trabalhar em diferentes relevos e que possa fazer uma exploração em diferentes terrenos, é um equipamento muito grande e caro, o que o torna um equipamento, geralmente, incapaz de ser replicado ou utilizado para estudo.

Isso fica evidente quando observam-se as sondas que atualmente estão sendo desenvolvidas e as que já estão em uso, todas custaram muito dinheiro e muitas pessoas atuaram em seu desenvolvimento. Claro, isso se dá, principalmente, pela magnitude e da necessidade do desenvolvimento tecnológico. No entanto, pela necessidade de muitos recursos tecnológicos e financeiros, muitos estudantes optam por nem cogitar a possibilidade de atuar nessa área.

Devido ao desenvolvimento ter envolvido um grande montante de recurso financeiro e tecnológico, as sondas são desenvolvidas com missões e objetivos bem claros e bem definidos. Essas missões podem ser desde missões voltadas à ciência, como por exemplo, medição dos campos magnéticos e elétricos do planeta, composição do solo, entre outras; podem existir aplicações voltadas à engenharia, como o comportamento do hardware utilizado, novos meios e protocolos de comunicação, entre outros [6].

Devido às diferentes atividades que podem ser realizadas e desenvolvidas por um rover, é esperado que ele possa ser utilizado pelos alunos como um meio de incentivo e apoio ao ensino/aprendizagem dos mesmos. Pensando nisso, a Agência Espacial Americana (NASA) resolveu disponibilizar o projeto de uma réplica do rover que atualmente está realizando uma missão em Marte, o Curiosity [7]. Além de iniciativas como a da agência americana, o incentivo pode ser dado, por exemplo, desenvolvendo trabalhos e estudos sobre protocolos de comunicação que podem ser utilizados, sistemas para ejeção e recuperação do satélite, telemetria e sensoriamento terrestre, entre inúmeros projetos de desenvolvimento científico e tecnológico que podem ser desenvolvidos com ele. Assim, poderia tornar o projeto de um rover mais barato e acessível.

Tendo isso em vista, este trabalho apresenta o desenvolvimento de um rover didático, que poderá ser utilizado para o estudo e desenvolvimento de novas tecnologias e, por meio desses estudos, incentivar e apoiar o processo de ensino/aprendizagem dos alunos. A proposta é que esse rover possa ser utilizado em unidades curriculares como redes de computadores, na utilização dos protocolos de comunicação; física; processamento de imagens, visão computacional e inteligência artificial, trabalhar com imagens de satélite para 
gerar conhecimento; sistemas operacionais e sistemas embarcados, para ver a melhor organização e arquitetura de hardware e software a ser utilizado; entre outras áreas.

\section{MOTIVAÇÃO}

Utilizar experimentos como ponto de partida para desenvolver a compreensão de conceitos vistos em sala de aula é uma tarefa considerada essencial, principalmente quando se considera conteúdos abstratos como os abordados em Física e Química, mas não exclusivamente dessas unidades curriculares.

Um meio de facilitar a compreensão dos conteúdos abstratos é por meio da experimentação. Essa experimentação pode ser realizada por simuladores ou por equipamentos reais, os quais os alunos podem tocar e sentir [8]. Um modo de aproximar os conhecimentos de unidades curriculares como Redes de Computadores, Sistemas Operacionais, Algoritmos, entre outras, é aplicando em um experimento real.

Porém, o experimento deve ser instigante e motivador para o aluno. Atualmente, a exploração espacial é um ponto que está em alta, vistas as inúmeras missões espaciais que ocorreram nos últimos anos e que estão previstas para as próximas.

Uma missão espacial geralmente envolve uma grande quantidade de equipamentos e, cada um, com uma finalidade diferente. Desde os lançadores até satélites e sondas que pousam em solo extraterrestre, todos esses equipamentos são extremamente caros e envolvem muita tecnologia e otimização [9]. Devido a grande tecnologia e a inovação envolvidas, esse tipo de equipamento torna-se bastante motivador aos alunos.

Assim, dentre todos esses equipamentos, esse trabalho teve como objetivo o desenvolvimento de uma sonda rover de exploração extraterrestre para fins didáticos.

O termo rover ficou mais popular após o uso do Curiosity no planeta Marte [10]. No entanto, esse não foi o primeiro veículo a pousar lá. Em 1997 o Pathfinder pousou em solo marciano e tinha como objetivo estudar a atmosfera marciana, seu clima, geologia e composição do solo. Com a popularidade e o objetivo desse tipo de equipamento, diversos outros rovers foram desenvolvidos e enviados à diferentes missões.

$\mathrm{O}$ uso desse tipo de equipamento em Marte, principalmente, se dá para exploração do planeta vermelho na esperança que ele possa ser habitável por humanos. Além disso, é possível que esse tipo de equipamento seja capaz de identificar organismos vivos, além de identificar os componentes do solo e da atmosfera marciana [11]. Essa exploração é importante para o avanço da ciência e pode ser utilizado como um estímulo ao desenvolvimento da criticidade, criatividade e dos conhecimentos de ciências em estudantes do ensino médio e do ensino superior.

\section{METODOLOGIA}

Para o desenvolvimento do rover didático, foi adotada uma metodologia de caráter exploratório, pois teve como objetivo o desenvolvimento de um sistema/equipamento para verificar a viabilidade de seu uso. Para isso, foram adotadas as seguintes etapas no desenvolvimento: (i) estudo sobre sondas e materiais que podem ser utilizados; (ii) definição da plataforma que será utilizada, se usará rodas, pernas ou esteiras; (iii) montagem da estrutura; (iv) definição e montagem dos motores e rodas, esteiras, pernas; (v) desenvolvimento do sistema de câmera; (vi) integração dos componentes; (vii) teste para subir e descer escadas; (viii) teste e validação em sala de aula.

Infelizmente, devido às restrições impostas para combater a pandemia que assolou o mundo em 2020, a etapa (viii) teste e validação em sala de aula não pôde ser realizadas.

\section{DESENVOLVIMENTO}

Durante a etapa de estudo a respeito de sondas e de materiais que podiam ser utilizados, foi encontrado o projeto disponibilizado pelo Laboratório de Propulsão ( Fet Lab Propulsion - JLP) da NASA [7] para montar uma réplica do Rover Curiosity, rover da NASA que está explorando a superfície marciana (Figura 1).

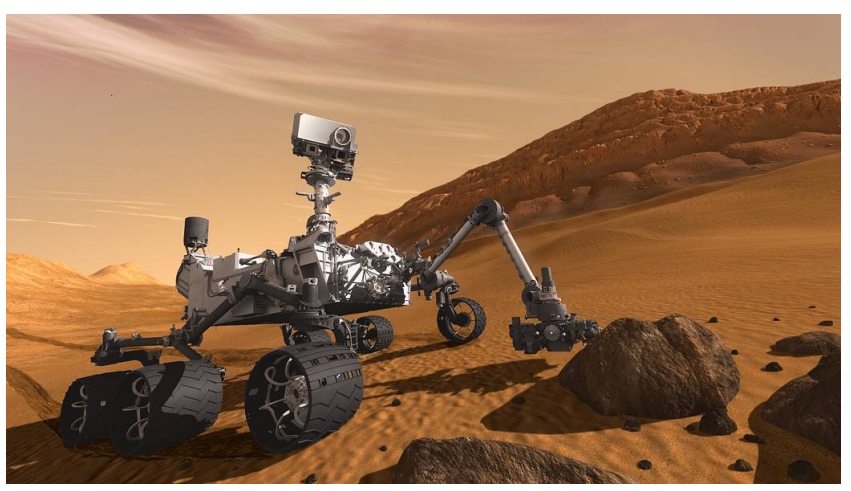

Figura 1: Rover Curiosity. Fonte: NASA [12]

Foi realizada uma análise do provável motivo do uso de rodas no Rover Curiosity em vez de esteiras. Esteiras são comumente utilizadas para movimentação dos robôs nas competições de robótica pelo mundo todo. Durante essa análise, percebeu-se que, devido a articulação entre duas rodas, isoladas de uma terceira, permite que o veículo tenha um ângulo de ataque superior ao de uma esteira, uma vez que essa articulação, ao ser acionada, é capaz de mover a roda do veículo para cima do obstáculo e também permite andar em terrenos irregulares. Assim, para que o rover seja capaz de se locomover em ambientes com diferentes obstáculos, as rodas são mais adequadas. Com isso, este projeto faz uso do mesmo padrão arquitetural e de movimentação que o Rover Curiosity, o arranjo de suspensão Rocker-bogie system [13] (Figura 2).

Após a definição da estrutura, iniciou-se a análise do material para montagem da estrutura do rover. No projeto do JLP a estrutura é toda de metal, o que dificulta sua manipulação e também a capacidade de replicabilidade com custo reduzido, uma vez que precisa equipamento especializado para corte e dobras de hastes, barras e chapas metálicas. Assim, optou-se pelo uso de tubos de PVC (Policloreto de Polivinila - Polyvinyl chloride) para montagem da estrutura. O PVC é um material de fácil manuseio, barato e encontrado em lojas de materiais de construção. Devido a essa versatilidade, optou-se pelo uso do PVC ao invés do uso de material metálico. O que permite também que o projeto possa ser replicado, sem a necessidade de diversos equipamentos para trabalhar com metais. 


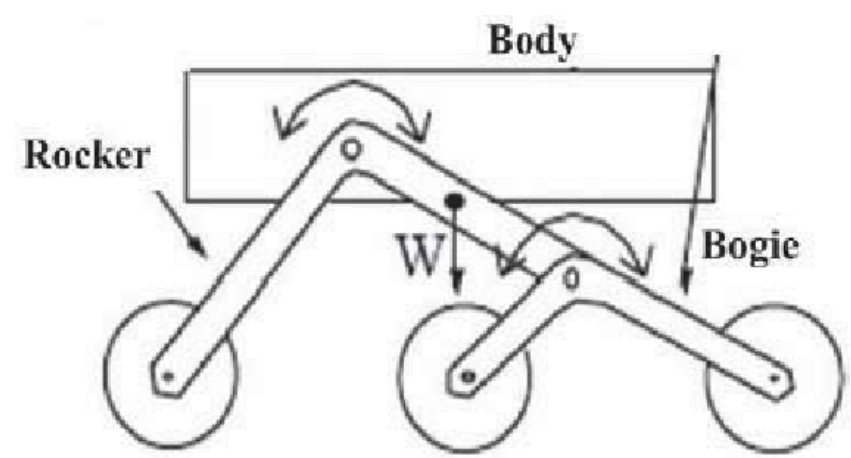

Figura 2: Sistema de suspensão Rocker. Fonte: [14]

Com a arquitetura definida e o material definido, iniciaram-se as buscas por algum projeto de rover ou robôs que fazem uso de rodas e são construídos de PVC. Nessa pesquisa foi encontrado o projeto Rocker Bogie Robot de [15], um rover controlado via joystick, feito de PVC, com a mesma arquitetura do rover Curiosity e também capaz de subir escadas. Esse projeto encontrado foi utilizado como referência no desenvolvimento do trabalho aqui apresentado. A Figura 3 apresenta uma imagem da estrutura de PVC montada.

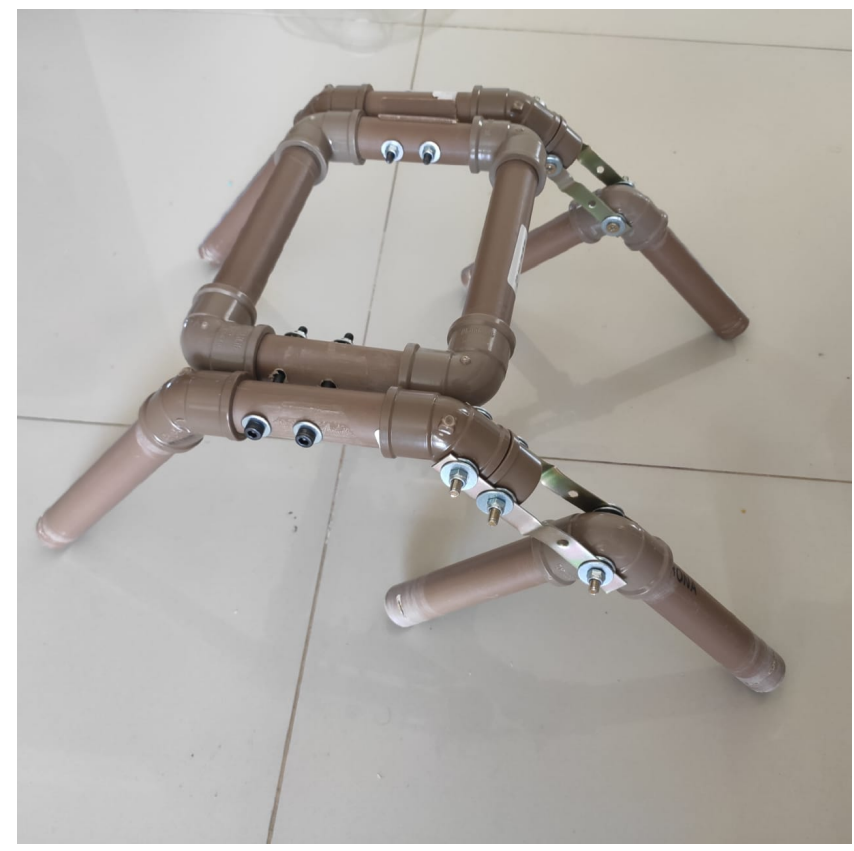

Figura 3: Estrutura de PVC montada com o sistema RockerBogie

No projeto apresentado por [15] são utilizados motores DC de alto torque e baixa rotação, torque de $15 \mathrm{~kg}-\mathrm{cm}$ e 30 rotações por minuto (RPM), além de fazer uso de pneus de $125 \mathrm{~mm}$ de diâmetro e rodas de $60 \mathrm{~mm}$ de diâmetro. Ambos difíceis de encontrar no mercado nacional brasileiro. Por isso, foi feita a busca por motores, rodas e pneus mais baratos e disponíveis no mercado nacional. Primeiro foi avaliado o uso do conjunto motor DC + roda disponível

\section{Tabela 1: Custos para aquisição do material}

\begin{tabular}{|l|l|l|l|}
\hline Qnt & Descrição & Unitário & Total \\
\hline 1 & Tubo de PVC $25 \mathrm{~mm}$ & $\mathrm{R} \$ 18,80$ & $\mathrm{R} \$ 18,80$ \\
\hline 4 & Joelhos $90^{\circ}$ PVC $25 \mathrm{~mm}$ & $\mathrm{R} \$ 0,70$ & $\mathrm{R} \$ 2,80$ \\
\hline 6 & Joelho $45^{\circ}$ PVC $25 \mathrm{~mm}$ & $\mathrm{R} \$ 1,50$ & $\mathrm{R} \$ 9,00$ \\
\hline 2 & Cap PVC $25 \mathrm{~mm}$ & $\mathrm{R} \$ 1,60$ & $\mathrm{R} \$ 3,20$ \\
\hline 6 & Abraçadeiras $25 \mathrm{~mm}$ & $\mathrm{R} \$ 2,80$ & $\mathrm{R} \$ 16,80$ \\
\hline 1 & Cola adesiva plástica & $\mathrm{R} \$ 20,00$ & $\mathrm{R} \$ 20,00$ \\
\hline 1 & Raspberry Pi Model B & $\mathrm{R} \$ 415,00$ & $\mathrm{R} \$ 415,00$ \\
\hline 1 & Câmera Raspberry Pi v2 8MP & $\mathrm{R} \$ 507,00$ & $\mathrm{R} \$ 507,00$ \\
\hline 6 & Motor DC 12v alto torque & $\mathrm{R} \$ 138,30$ & $\mathrm{R} \$ 830,00$ \\
\hline 6 & Roda com pneu 125 mm + ATV & $\mathrm{R} \$ 197,00$ & $\mathrm{R} \$ 1.182,00$ \\
\hline 6 & Acoplador interno roda 6.1mm & $\mathrm{R} \$ 98,00$ & $\mathrm{R} \$ 588,00$ \\
\hline 1 & Controle PS2 com driver motor & $\mathrm{R} \$ 497,00$ & $\mathrm{R} \$ 497,00$ \\
\hline 1 & Bateria DC 12v & $\mathrm{R} \$ 112,00$ & $\mathrm{R} \$ 112,00$ \\
\hline $\mathrm{X}$ & Parafusos, porcas e arruelas & $\mathrm{R} \$ 30,00$ & $\mathrm{R} \$ 30,00$ \\
\hline & & $\mathrm{TOTAL}$ & $\mathrm{R} \$ 4.231,60$ \\
\hline
\end{tabular}

em kits de robóticas, mas esse motor não possui torque suficiente para mover toda a estrutura e forma controlada. Assim, foi necessário buscar um motor mais próximo ao apresentado no projeto original, motor DC $12 \mathrm{~V}$ de $22 \mathrm{RPM}$ e torque de $13,5 \mathrm{~kg}-\mathrm{cm}$, modelo JGB37-520.

Foram utilizados motores de alto torque devido ao peso final do rover e a necessidade de ultrapassar obstáculos. Um motor com baixo torque não fornece energia suficiente para que as rodas possam subir obstáculos grandes. Já as rodas, optou-se pelo uso de rodas grandes e robustas, pois os motores possuem torque suficiente para sua movimentação e, obstáculos pequenos são facilmente ultrapassados.

A fonte de alimentação foi utilizada uma bateria com tensão suficiente para alimentação dos motores, uma bateria selada de chumbo-ácido de $12 \mathrm{~V}$, presa na estrutura do rover. Já para o controlar sua movimentação, para a sua primeira versão, foi utilizado um controle de console PlayStation 2 (PS2) sem fio, comunicando com um módulo receptor sem fio e um driver para motor. Assim, ao acionar o joystick do controle, o rover efetua sua movimentação. Como trabalho futuro se tem a proposta do uso de um Raspberry Pi para efetuar o controle dos motores.

Além disso, o rover precisa efetuar uma missão, para a primeira versão, apenas observar o campo em que ele se encontra. Para isso, foi utilizado uma câmera e um Raspberry Pi. Com isso, o Raspberry Pi se conecta a uma rede WiFi e cria um servidor de vídeo. Ao acessar este servidor, é possível visualizar o vídeo capturado pela câmera.

Em suma, o rover é baseado no projeto do rover Curiosity da NASA [7], e também no projeto de [15], feito de PVC, com seis rodas de $125 \mathrm{~mm}$ de diâmetro, seis motores DC $12 \mathrm{~V}$ com torque de $13,5 \mathrm{~kg}$-cm e 22RPM, possui uma câmera e um servidor de vídeo para transmissão remota. O custo para montar esse projeto foi cerca de $\mathrm{R} \$ 4.231,60$, conforme apresentado na Tabela 1. 


\section{TESTES}

Para os testes de funcionamento do rover foram elencados dois roteiros, (1) mover para frente, para trás e fazer curvas para ambos os lados; (2) subir pelo menos 1 degrau de escada. Infelizmente devido às restrições impostas para tentar combater o avanço da pandemia do Covid-19, não foi possível a realização dos testes em sala de aula, para que os alunos pudessem realizar experimentos e programar o rover.

No roteiro 1 o rover realizou todos os movimentos conforme previsto. Já para o roteiro 2 ele foi capaz de subir obstáculos de até $15 \mathrm{~cm}$ de altura. A Figura 4 apresenta o rover desenvolvido subindo um obstáculo de $15 \mathrm{~cm}$ de altura, um step. Durante esse teste foi observado que as chapas metálicas utilizadas como articulação do Bogie (vide Figura 2) era muito flexíveis e sofriam torção ao tentar passar por obstáculos com mais de $13 \mathrm{~cm}$ de altura. Essa correção será feita nas atualizações e novas versões do rover.

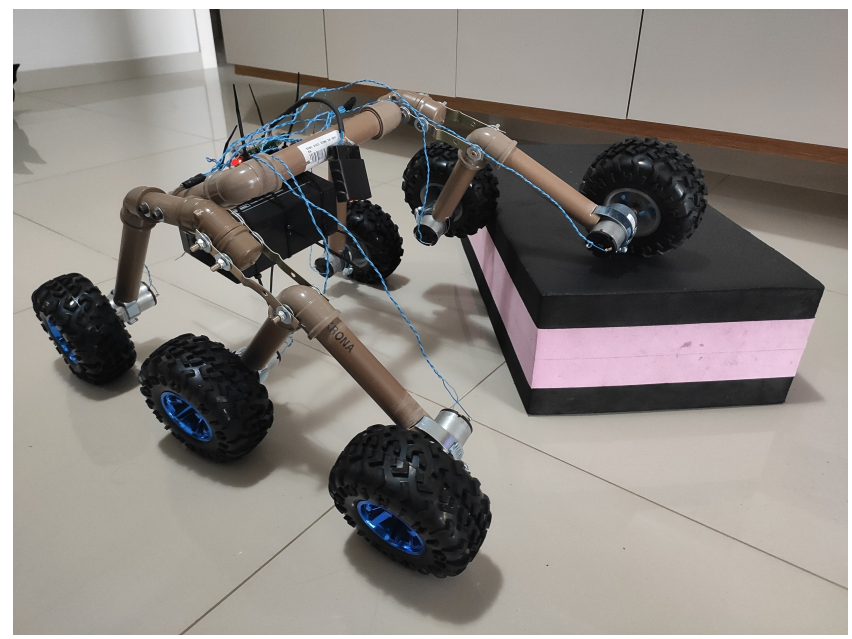

Figura 4: Rover desenvolvido subindo obstáculo de $15 \mathrm{~cm}$ de altura

Está previsto o uso do rover em unidades curriculares como redes de comunicação, para avaliar os diferentes protocolos que podem ser utilizados e; física e geografia, simular e explicar aos estudantes como funciona uma exploração espacial, como pode ser realizado o controle remoto de uma sonda e como são obtidas as imagens. Porém, por ser um objeto que possui um impacto visual, é necessário que hajam atividades presenciais para sua utilização.

\section{CONSIDERAÇÕES FINAIS}

A exploração espacial está em amplo avanço e é necessário que cada vez mais estudantes estejam interessados em aprender as ciências necessárias para desenvolver as tecnologias aeroespaciais. Pensando em aproximar mais a comunidade aos conceitos de ciências e também de novas tecnologias, esse trabalho apresentou o desenvolvimento de uma sonda rover baseada no projeto do Rover Curiosity [7], sonda que está explorando e realizando descobertas no território de marciano.

Essa foi a primeira versão do projeto, e foi influenciado pelo projeto apresentado por [15], um rover baseado no Curiosity, feito de tubos de PVC, com seis rodas grandes e motores de alto torque, controlado remotamente por um joystick e transmitindo a imagem via $\mathrm{WiFi}$, tudo alimentado por uma bateria.

Nas próximas atualizações do rover está previsto o uso de processamento de imagens para identificar algum padrão, realizar o controle e movimentação remota via placa Raspberry Pi e de forma autônoma, melhorar o acabamento da estrutura de PVC e, transmitir o vídeo a longas distâncias. Também está previsto o teste e avaliação do rover em ambiente escolar para instigar os estudantes a ingressarem nas áreas das STEAMs (Science, Technology, Engineering, Arts and Mathematics).

Com essas atualizações previstas, além das unidades curriculares já comentadas, poderão também ser trabalhados conceitos de programação, controle e automação, programação concorrente, entre outras. Além de abrir um leque maior de pesquisa que pode ser desenvolvida em cima desse rover.

\section{ACKNOWLEDGMENTS}

Projeto desenvolvido pelo GFIG - Grupo de Foguetes do IFSC Gaspar e financiado pelo EDITAL EDITAL N ${ }^{\circ}$ 42/2019 - PROPPI Programa de Apoio ao Fortalecimento de Grupos de Pesquisa e Núcleos de Atividades do Câmpus Gaspar.

\section{REFERÊNCIAS}

[1] Redação Galileu. Robô britânico será enviado ao espaço para explorar a lua em 2021, 2019. URL https://revistagalileu.globo.com/Ciencia/Espaco/noticia/2019/ 10/robo-britanico-sera-enviado-ao-espaco-para-explorar-lua-em-2021.html.

[2] Redação Galileu. Startup israelense abandona planos de ir para a lua, 2019. URL https://revistagalileu.globo.com/Ciencia/Espaco/noticia/2019/06/startupisraelense-abandona-planos-de-ir-para-lua.html.

[3] Redação Galileu. Nasa projeta helicóptero para sobrevoar marte em 2021, 2018. URL https://revistagalileu.globo.com/Ciencia/noticia/2018/05/nasa-projetahelicoptero-para-sobrevoar-marte-em-2021.html.

[4] Redação do Site Inovação Tecnológica. Sonda osiris-rex captura amostras do asteroide bennu, 2020. URL https://www.inovacaotecnologica.com.br/noticias/ noticia.php?artigo=sonda- osiris-rex-captura-amostras-asteroide-bennu.

[5] AEROJR. O que são sondas espaciais?, 2019. URL https://aerojr.com/blog/o-quesao-sondas-espaciais/.

[6] S. Antunes. DIY Satellite Platforms. Oreilly and Associate Series. O'Reilly, 2012. ISBN 9781449310608.

[7] NASA JPL. Jpl's open source build-it-yourself rover, 20? URL https:// opensourcerover.jpl.nasa.gov/.

[8] L. R. P. Rauta and A. M. da R. Fernandes. Ferramenta computacional de apoio ao ensino/aprendizado de farmacologia. In Nuevas Ideas en Informática Educativa - Memorias del XIX Congresso Internacional Informatica Educativa - TISE 2014, volume 1, pages 246-256, Fortaleza, Brazil, 2014. URL http://www.tise.cl/volumen10/TISE2014/tise2014_submission_211.pdf.

[9] J. K. Erickson. Living the dream - an overview of the mars exploration project. IEEE Robotics Automation Magazine, 13(2):12-18, 2006. doi: 10.1109/MRA.2006. 1638011.

[10] Redação Galileu. Curiosity revela detalhes sobre lago em marte há 3 bilhões de anos, 2020. URL https://revistagalileu.globo.com/Ciencia/Espaco/noticia/2020/05/ curiosity-revela-detalhes-sobre-lago-em-marte-ha-3-bilhoes-de-anos.html.

[11] Balakrishnan Shankar, Ganesha Udupa, Praveen Basil, Aswath Suresh, Sreekuttan Kalathil, C. Hariprasad, and Ragesh Ramachandran. Design and development of an intelligent rover for mars exploration(updated). 012015.

[12] NASA. Curiosity - the next mars rover, 2011. URL https://www.nasa.gov/mission pages/msl/multimedia/gallery/pia14156.html.

[13] Abdullah Cihan Ozdemir et al. Effect of rocker double lambda mechanism on mobile robot. Journal of Young Investigators, 37:51-56, 2020.

[14] A. A. Rafiq, M. Yusuf, and Pujono. Implementation of digital image processing using ni myrio and arduino mega 2560 as controller on rover bogie robot. In 2018 International Conference on Applied Science and Technology (iCAST), pages 210-215, 2018. doi: 10.1109/iCAST1.2018.8751506.

[15] N. Khambhala. Rocker bogie robot "stair climbing car", 2017. URL https:// navinkhambhala.com/rocker-bogie-robot. 